\title{
The challenges and opportunities of global neurosurgery in East Africa: the Neurosurgery Education and Development model
}

\author{
Andreas Leidinger, MD, ${ }^{1,2}$ Pablo Extremera, MD, ${ }^{3}$ Eliana E. Kim, BA, ${ }^{2}$ \\ Mahmood M. Qureshi, FRCSEd(SN), ${ }^{4}$ Paul H. Young, MD, PhD, ${ }^{5}$ and José Piquer, MD, PhD 1,6 \\ ${ }^{1}$ Neurosurgery Education and Development Foundation, Valencia, Spain; ${ }^{2}$ Neurosurgery Education and Development Institute, \\ Mnazi Mmoja Hospital, Stonetown, Zanzibar, Tanzania; ${ }^{3}$ Hospital Universitario de La Paz, Madrid, Spain; ${ }^{4}$ Aga Khan University, \\ Nairobi, Kenya; ${ }^{5}$ Section of Neurosurgery, Department of Surgery, St. Louis University, St. Louis, Missouri; and ${ }^{6}$ Hospital \\ Universitario de la Ribera, Alzira, Valencia, Spain
}

OBJECTIVE The objective of this study was to describe the experience of a volunteering neurosurgeon during an 18week stay at the Neurosurgery Education and Development (NED) Institute and to report the general situation regarding the development of neurosurgery in Zanzibar, identifying the challenges and opportunities and explaining the NED Foundation's model for safe practice and sustainability.

METHODS The NED Foundation deployed the volunteer neurosurgeon coordinator (NC) for an 18-week stay at the NED Institute at the Mnazi Mmoja Hospital, Stonetown, Zanzibar. The main roles of the NC were as follows: management of patients, reinforcement of weekly academic activities, coordination of international surgical camps, and identification of opportunities for improvement. The improvement opportunities were categorized as clinical, administrative, and sociocultural and were based on observations made by the NC as well as on interviews with local doctors, administrators, and government officials.

RESULTS During the 18 -week period, the NC visited 460 patients and performed 85 surgical procedures. Four surgical camps were coordinated on-site. Academic activities were conducted weekly. The most significant challenges encountered were an intense workload, deficient infrastructure, lack of self-confidence among local physicians, deficiencies in technical support and repairs of broken equipment, and lack of guidelines. Through a series of interviews, the sociocultural factors influencing the NED Foundation's intervention were determined. Factors identified for success were the activity of neurosurgical societies in East Africa; structured pan-African neurosurgical training; the support of the Foundation for International Education in Neurological Surgery (FIENS) and the College of Surgeons of East, Central and Southern Africa (COSECSA); motivated personnel; and the Revolutionary Government of Zanzibar's willingness to collaborate with the NED Foundation.

CONCLUSIONS International collaboration programs should balance local challenges and opportunities in order to effectively promote the development of neurosurgery in East Africa. Support and endorsement should be sought to harness shared resources and experience. Determining the caregiving and educational objectives within the logistic, administrative, social, and cultural framework of the target hospital is paramount to success.

https://thejns.org/doi/abs/10.3171/2018.7.FOCUS18287

KEYWORDS neurosurgery; East Africa; low- and middle-income countries; sustainability; development

$\mathrm{S}$ INCE 2014, the World Bank has exhorted the medical community to design a global strategy that promotes universal access to basic surgical services. ${ }^{4}$ A report from the Lancet Commission on Global Surgery (Global Surgery 2030) concluded that the progressive growth of surgery in low- and middle-income countries improves general health and promotes economic growth. ${ }^{14}$ The development of surgical subspecialties, including neurosurgery, and their widespread availability are among the main objectives of the international community for global surgery. ${ }^{22}$

ABBREVIATIONS COSECSA = College of Surgeons of East, Central and Southern Africa; ED = Emergency Department; FIENS = Foundation for International Education in Neurological Surgery; ICP = international collaboration program; ICU = intensive care unit; ISC = international surgical camp; NC = neurosurgeon coordinator; NED = Neurosurgery Education and Development; NEDF = NED Foundation; NEDI = NED Institute; MMH = Mnazi Mmoja Hospital; RGZ = Revolutionary Government of Zanzibar; $\mathrm{TBI}=$ traumatic brain injury; WFNS = World Federation of Neurosurgical Societies; WHO = World Health Organization.

SUBMITTED June 1, 2018. ACCEPTED July 10, 2018.

INCLUDE WHEN CITING DOI: 10.3171/2018.7.FOCUS18287. 
Many factors hamper the structured growth of surgical subspecialties in East Africa: geographic isolation of many communities, bureaucratic resistance, political instability, constant socioeconomic changes, high rates of poverty and illiteracy, and rapidly growing population. ${ }^{4,5}$ Undoubtedly, the complex situation in East Africa hinders the growth of neurosurgery, ${ }^{7}$ but this reality should not be perceived as an insurmountable obstacle; rather, it is an inevitable challenge that African physicians and global surgeons must tackle in the 21 st century. ${ }^{14,17}$

Several international collaboration programs (ICPs), most of which are directed from less developed countries, have partnered with African hospitals using diverse methodologies toward training African specialists. ${ }^{10,15,18,19}$ These organizations have been clustered around institutions such as the World Federation of Neurosurgical Societies (WFNS; https://www.wfns.org/menu/11/trainingcenters-fellowship) and the Foundation for International Education in Neurological Surgery (FIENS; http://www. fiens.org), which, in association with the Lancet Commission on Global Surgery ${ }^{14}$ and the World Health Organization (WHO), act as global coordinators and leaders of ICPs in Africa. ${ }^{6}$

Among these ICPs, the Neurosurgery Education and Development (NED) Foundation (NEDF) has conducted surgical camps (e.g., neuroendoscopy unit) in East Africa since 2006. ${ }^{18}$ The two main purposes of the NEDF are to provide neurosurgical care in East Africa and to improve neurosurgical education for physicians and nurses. Since the establishment of the NED Institute (NEDI) in Zanzibar in $2014,{ }^{10}$ the NEDF has focused on consolidating an autonomous neurosurgical center. ${ }^{20,22}$ The NEDI hosts monthly surgical camps, organizes international academic activities, and is a potential center for training African residents.

The main objective of this study is to describe the clinical experience obtained by a volunteer neurosurgeon during an 18-week stay at the NEDI and to describe the most relevant opportunities for improvement. Finally, we wish to describe the economic, social, and cultural situation of medical practice in Zanzibar and its effects on ICPs.

\section{Methods}

The volunteer was recruited by the NEDF in 2017. He performed as a neurosurgeon coordinator (NC) during an 18-week stay from November 2017 to March 2018 at the NEDI. The selection process consisted of a series of interviews with Dr. José Piquer, the current president of the NEDF.

\section{Objectives of the NC}

The main objectives of the NC were as follows: management of patients, reinforcement of weekly academic activities, coordination of international surgical camps (ISCs), and identification of opportunities for improvement. The improvement opportunities were categorized as clinical, administrative, and sociocultural.

Management of patients involved performing scheduled surgical procedures twice per week, daily visitation of admitted patients, and medical management in the neurosurgical ward and intensive care unit (ICU). The NC was available 24/7 for telephonic consultations and emergency surgeries.

The NC served as organizer and field coordinator of several ISCs. The NC also kept in close communication with the ICU and local rehabilitative care teams, with which the NEDF has also established collaboration.

Finally, the NC interviewed local doctors, administrators, and government officials, such as two consecutive health ministers (Mahmoud Kombo Thabit and Hamad Rashid Mohammed). During these interviews, important social and cultural aspects of the current health situation in Zanzibar were discussed and recorded.

\section{The Mnazi Mmoja Hospital and the NEDI}

The NEDI is localized within the perimeter of the Mnazi Mmoja Hospital (MMH) in the city of Stonetown, Zanzibar. The MMH is the single tertiary care referral hospital in the Zanzibar archipelago (consisting of two main islands, Unguja and Pemba, and numerous other minor scarcely populated islets), which has 1.8 million inhabitants. ${ }^{16}$ Currently, the NEDI acts as the single neurosurgical care provider in Zanzibar. As of April 2018, the NEDI is staffed by one trained local neurosurgeon, one fast-track trained local medical officer, and two other off-site surgeons who are receiving formal neurosurgical training abroad. The NEDI is codirected by Dr. Mohammed A. Haji, a local coordinator, and Dr. José Piquer, the president of the NEDF.

The NEDI has 24 inpatient beds, two outpatient clinic consultation rooms, one administrative office, and two dedicated neurosurgical theaters equipped with flexible beds, air conditioning, anesthesiology ventilators, and cardiac monitors, all provided by the NEDF. The NEDI's surgical storage holds over 30 boxes of surgical material, including all the basic materials to perform neurosurgical procedures.

\section{Results \\ Clinical Results}

During the study time frame, approximately 460 patients were seen by the $\mathrm{NC}$ in the outpatient clinic and 85 surgical procedures were performed (Fig. 1 and Table 1). Conducted academic activities included daily clinical case presentations and radiological discussions and a weekly clinical session in which relevant clinical topics were discussed with local medics and nurses. Table 2 summarizes the academic talks conducted. Four ISCs were arranged by the NEDF (three groups from Spain and one from Germany) during the NC's stay. The surgical teams were composed of 5-10 healthcare professionals, including neurosurgeons, anesthesiologists, intensivists, surgical nurses, and physiotherapists. Surgical camps were composed of one clinical day and five surgical days. For some days, both theaters were simultaneously active. Academic activities such as morning lectures on relevant topics were also conducted by foreign faculty. Figure 2 summarizes the flux of international volunteers at NEDI, their time of stay, and their field of expertise.

\section{Clinical Challenges \\ Workload}

A high volume of patients exceeds the capacity of lo- 


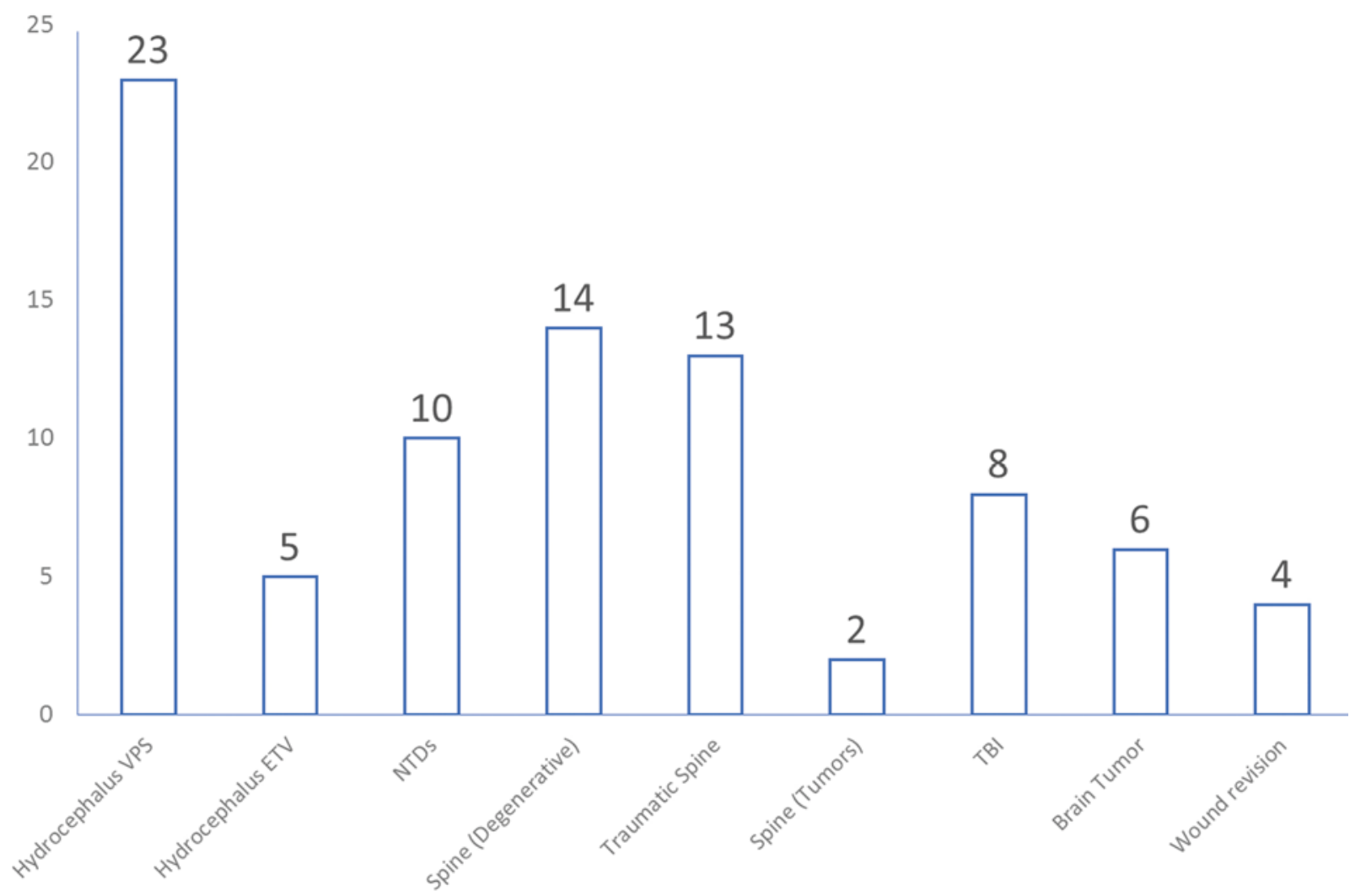

FIG. 1. Details of surgical activity. ETV = endoscopic third ventriculostomy; NTD = neural tube defect; VPS = ventriculoperitoneal shunting. The $y$-axis represents the number of patients.

cal physicians and surgeons at the NEDI and MMH, generating long waiting lists. Only one anesthesiologist with experience in neurosurgical and pediatric anesthesia is available at MMH. Therefore, surgery at the NEDI is performed only twice per week. Alleviating the intense workload is one of the reasons the NEDI still hosts monthly ISCs. Furthermore, a high percentage of the daily workload at MMH and NEDI is administrative or bureaucratic in nature, which restricts the amount of time local physicians can dedicate to clinical care, training, and research.

\section{Infrastructure and Healthcare}

The NEDI is functionally equipped and in better condition than most other hospitals in East Africa. Moreover, the Revolutionary Government of Zanzibar (RGZ) provides financial coverage of some medical expenses, such as CT scan and MRI acquisitions, for all patients. Still, there is a constant shortage of basic medicine and materials, most commonly film for radiograph development, laboratory reactants, gauze, sterile gloves, and plaster. Essential supplies such as oxygen, electricity, and water are unsteady and pose a potential threat to the performance of healthcare teams and ISCs. Figure 3 summarizes the availability of basic resources during the study time frame.

\section{Self-Confidence and the Value of Knowledge}

Local medics and nurses are generally not confident regarding their own knowledge and expertise. Surgeons usually doubt the surgical indications and consult the NC before asserting their own medical judgment. Because of the intense workload and lack of resources at the NEDI, local personnel tend to adopt a work approach focused on individual patients, rather than understanding the general management algorithms and their clinical rationale. In this regard, theoretical knowledge that has no immediate use to improve the patient's condition is often perceived as less relevant. Hence, patient care is usually provided in response to specific symptoms and not to clinical syn-

TABLE 1. Details of surgical activity

\begin{tabular}{lccccc}
\hline \multicolumn{1}{c}{ Parameter } & Nov 2017 & Dec 2017 & Jan 2018 & Feb 2018 & Total \\
\hline Surgical procedures & 32 & 11 & 24 & 18 & 85 \\
\hline Complications & $9.4 \%(3 / 32)$ & $18.2 \%(2 / 11)$ & $8.3 \%(2 / 24)$ & $11 \%(2 / 18)$ & $10.6 \%(9 / 85)$ \\
\hline Mortality & $0 \%$ & $0 \%$ & $8.3 \%(2 / 24)$ & $11 \%(2 / 18)$ & $4.7 \%(4 / 85)$ \\
\hline Re-interventions & $0 \%$ & $9.1 \%(1 / 11)$ & $4.2 \%(1 / 24)$ & $11 \%(2 / 18)$ & $4.7 \%(4 / 85)$ \\
\hline
\end{tabular}


TABLE 2. Conducted oral presentations

\begin{tabular}{clcl}
\hline Date & Academic Talks & No. of Participants & Speaker \\
\hline $11 / 01 / 17$ & "TBI: medical management" & 6 & Leidinger, MD \\
\hline $11 / 17 / 17$ & "How to read a brain CT scan" & 8 & Leidinger, MD \\
\hline $11 / 24 / 17$ & "Initial assesment \& management of low-back pain" & 13 & Leidinger, MD \\
\hline $12 / 06 / 17$ & $\begin{array}{c}\text { "Neurotrauma workshop," full-day seminar covering TBI \& } \\
\text { traumatic spine }\end{array}$ & 38 & $\begin{array}{c}\text { Leidinger, MD; Idrissa, MD; } \\
\text { Salgado, MD; Buxeda, MD }\end{array}$ \\
\hline $01 / 04 / 18$ & "Acute hydrocephalus: a neurosurgical urgency" & 6 & Leidinger, MD \\
\hline $02 / 10 / 18$ & "Selected neuroradiology cases (TBI)" & 11 & Leidinger, MD \\
\hline $02 / 17 / 18$ & "Selected neuroradiology cases (hydrocephalus)" & 11 & Idrissa, MD \\
\hline $02 / 24 / 18$ & "Tuberculosis spine: medical \& surgical management" & 7 & Leidinger, MD \\
\hline $03 / 03 / 18$ & "External ventricular/lumbar drainage management" & 8 & Leidinger, MD \\
\hline $03 / 10 / 18$ & "Hydrocephalus in Zanzibar: our early experiences" & 28 & Leidinger, MD \\
\hline
\end{tabular}

dromes. For example, an admitted patient who suffered acute spinal trauma with a clear sensory level and a second patient admitted as an outpatient for a scheduled lumbar canal stenosis decompression, both presenting with bilateral paresthesia, could be given equal surgical priority since both present with similar symptomatology.

To address this issue, a daily clinicoradiological discussion was introduced. Needed tools for further training were provided, such as web resources, peer-reviewed papers, and access to online webinars. Active participation of the local NEDI personnel during these academic activities seemed to increase their confidence in their daily han- dlings, such as the in-ward management of drip chambers of external ventricular drains or passive mobilization of bedridden patients.

\section{Time and Priority}

Local practitioners have a different understanding of the value of time. This is especially evident in the torpid management of emergencies. In general, delays in patient referral to the Emergency Department (ED), its triage, and its initial assessment are the locally accepted standard. Finally, the acquisition of diagnostic neuroimaging can also delay treatment even further. Local practitioners frequent-

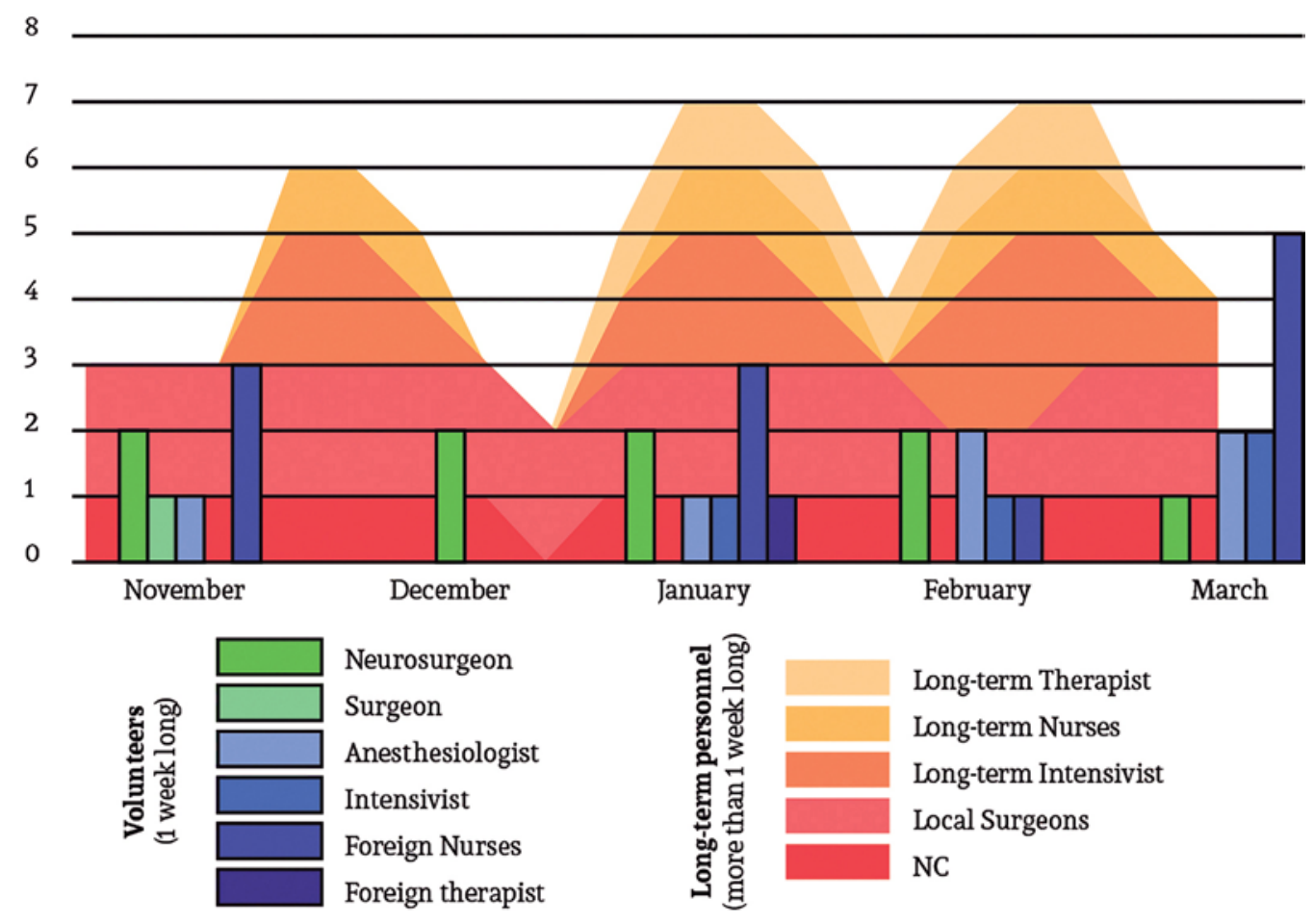

FIG. 2. Flux of foreign personnel during the 18-week study period. Bars represent the numbers of volunteers attending to surgical camps (1 week long). The stacked graphic area in the background represents all personnel staying longer than 1 week at the NEDI. Local surgeons were included in this graphic, but local nurses (19) and orderlies (6) were excluded, for scaling purposes. 


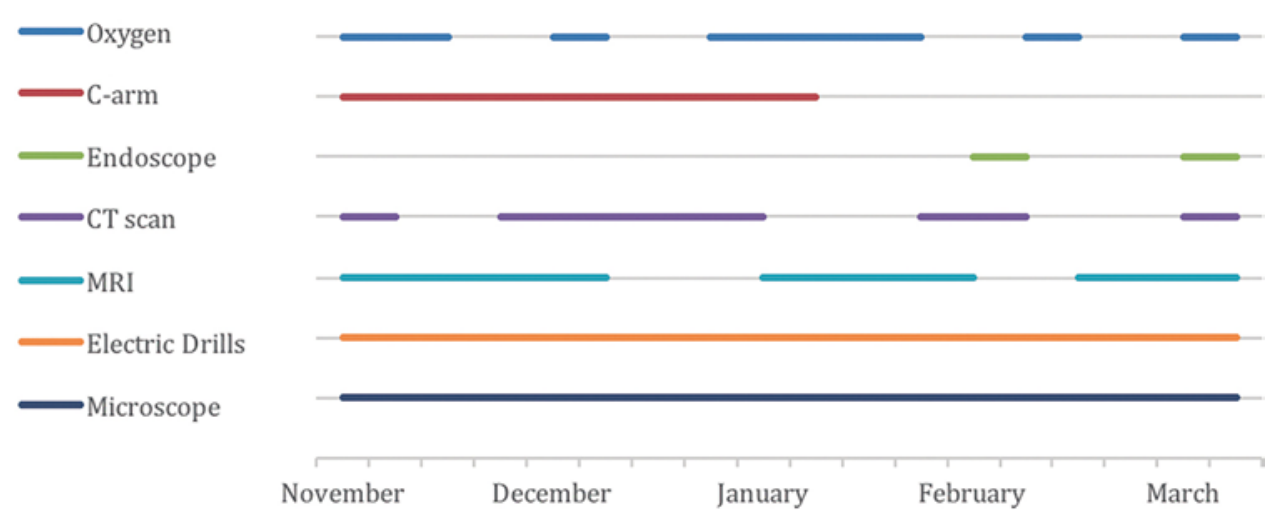

FIG. 3. Availability of basic resources at NEDI-MMH during the study period. Unavailability was attributable to equipment mishaps or lack of supply and is expressed as a discontinuation of each colored line.

ly suffer such delays and cannot expedite the slow patient circuit through the ED.

The constant need for reducing the waiting queue at the ED compels the medics to attend first to patients with less complex pathology who do not require labs or images in order to quickly reduce the volume of visitors. Critical patients are not prioritized. Furthermore, this practice motivates patients to visit a tertiary care hospital for minor ailments, contributing to the centralization of healthcare and the saturation of MMH and NEDI capacities.

\section{Integration Between Healthcare Teams}

The management of patients requiring more than one specialist, such as trauma patients, is hampered by scant communication between medical teams. For example, a severe traumatic brain injury (TBI) patient could be left waiting in the general wards prior to heart monitoring or neuroimaging acquisition, expecting the on-call intensivist to consider the patient worthy of ICU admission or not. Such an assessment could take from hours to several days. During his stay, the NC promoted a weekly session with intensivists, conducted visitation of ICU patients three days per week, and arranged a visit by the rehabilitation team at the NEDI once a week. Overall, better communication was achieved regarding individual patients, and initiatives for future cooperation between those medical teams arose. Other clinical improvement opportunities were identified during this study. Table 3 summarizes these opportunities.

\section{Administrative Challenges}

\section{Technical Service and Repairs}

Much of the medical equipment available at MMH and NEDI come from donations or the purchase of secondhand equipment thanks to private efforts under the direction of the NEDF's board committee. The hospital has no established relationship with the official suppliers. Hence, equipment tends to deteriorate faster through multiple interventions of the inexperienced local technicians. In several locations, equipment repair requires the intervention of out-sourced official providers that mostly fly in from Kenya and South Africa. The NEDF is constantly trying to reduce these delays by donating needed hardware and networking with different providers to repair broken equipment.

\section{Document and Information Administration}

Recently, the development of telecommunications in East Africa has allowed the massive diffusion of smartphones and tablets. In a context with limited access to desktop computers and storage, we recommend utilizing digital clouds for documents and formularies to be stored and shared. Furthermore, many mobile software and smartphone applications are specially designed for patient recording. The local team is interested in such resources and collaborated with the organization of digital folders and files.

\section{Sociocultural Challenges}

It is essential to recognize that the perceived role of physicians, the concept of well-being, and the value and quality of life vary across different cultures and social groups.

\section{General Layout of the Zanzibar Archipelago}

The Zanzibar archipelago is among the most diverse societies in East Africa. ${ }^{3}$ While hosting diverse ethnicities and traditions, social cohesion is maintained around Islam, the Swahili language, and a strong community identity. ${ }^{1,13,21}$ The coexistence of numerous minorities is maintained through strict courtesy rules and specific social spaces, tacitly defined for each collective of people. ${ }^{3}$ A Muslim patriarchal hierarchy prevails and influences almost all aspects of daily life. ${ }^{13}$ The majority of the population lives in rural areas where everyone plays a vital role in their community and their personal success is a function of community value. This largely explains the evident rejection and hiding of handicapped or diseased relatives who contribute less to communal tasks. ${ }^{18}$

In this strong community-oriented society, Zanzibari men engage in extensive heated greetings, long conversations, and a general attitude of service toward their peers. They use folk expressions such as "pole pole" or "Hakuna Matata," which mean "slowly, slowly" and "there are no problems" in Swahili, to urge their younger peers to worry 
TABLE 3. Opportunities and suggestions for improvement

\begin{tabular}{|c|c|c|}
\hline Opportunity for Improvement & Proposal & Current Status \\
\hline High patient load & Recruit additional surgeons; adhere to scheduled consultation days & Suggestion \\
\hline Repairs \& technical service & Keep contact w/ original providers to guarantee constant technical support & Ongoing initiative \\
\hline Personnel self-confidence & $\begin{array}{l}\text { Clinical meeting to discuss patients \& neuroimages (daily); dedicated lecture } \\
\text { (weekly); stimulate locals to participate during lectures }\end{array}$ & Ongoing initiative \\
\hline Wrong sense of timing & $\begin{array}{l}\text { Academic activities targeting ED \& ICU doctors; define specific algorithms for } \\
\text { TBI \& traumatic spine management }\end{array}$ & Completed, ongoing initiative \\
\hline $\begin{array}{l}\text { Deficient integration btwn medical } \\
\text { teams }\end{array}$ & $\begin{array}{l}\text { Address the problem w/ mobile-phone group chats to share clinically relevant } \\
\text { information \& images; invite intensivists to our morning meeting, weekly }\end{array}$ & Completed \\
\hline Hand hygiene (during rounds) & Provide hand soap \& alcohol gel at every ward in the NEDI & Completed \\
\hline $\begin{array}{l}\text { Missing exploration beds in external } \\
\text { consults }\end{array}$ & $\begin{array}{l}\text { Received private donation of } 2 \text { exploration beds; rearranged the furniture at } \\
\text { the consultation unit }\end{array}$ & Completed \\
\hline Lack of physiotherapy at NED & $\begin{array}{l}\text { Collaboration w/ local physiotherapists; invite them to participate at weekly } \\
\text { academic meeting; invite physiotherapist to participate in rounds, weekly }\end{array}$ & Ongoing initiative \\
\hline Personnel shortage: anesthesiologist & Search for external anesthesiologists to work part-time at NEDI & Suggestion \\
\hline $\begin{array}{l}\text { Early detection of warning signs in } \\
\text { postop patients }\end{array}$ & Clear explanation of warning signs after each surgery to nurse in charge & Completed, ongoing initiative \\
\hline Cleanliness of the NEDI & Daily cleaning \& supervision & Completed, ongoing initiative \\
\hline $\begin{array}{l}\text { Participate actively during surgical } \\
\text { procedures }\end{array}$ & $\begin{array}{l}\text { Detailed explanation of surgical procedure \& critical moments; everyone } \\
\text { should feel responsible }\end{array}$ & Ongoing initiative \\
\hline $\begin{array}{l}\text { Absence of neurosurgical education } \\
\text { in peripheral primary hospitals }\end{array}$ & $\begin{array}{l}\text { Organized "Neurosurgical warning signs," a talk in } 19 \text { peripheral primary } \\
\text { health centers in Zanzibar, including Pemba }\end{array}$ & Completed \\
\hline
\end{tabular}

Most relevant improvement opportunities were summarized. The Current Status column reflects whether the proposals are "suggestions" being considered by the NEDF, "ongoing initiatives" that were started during the NC's stay at the NEDI and need to be carried on, or "completed" if specific interventions were conducted during the stay of the NC at the NEDI.

only about current happenings or only those that immediately affect their communities or families, not thinking about tomorrow. ${ }^{21}$

Medical professionals are no exception to this laidback aspect of Zanzibari culture. They will not stress over deadlines or the many challenges to address. According to several physicians in $\mathrm{MMH}$, new opportunities from the globalized world are sometimes perceived as alien and too "fast-paced" for the Zanzibari standard of living.

\section{Role of Physicians}

In Zanzibar, the popular understanding of health and disease still calls upon magic, mystic, and religious concepts. ${ }^{2}$ The understanding of anatomical or physiological concepts by the local villagers is minimal. Consequently, the doctor-patient relationship is predominantly unidirectional, vertical, and strongly paternalistic. Doctors are expected to conduct themselves under strict social norms, and their medical skills and knowledge are rarely questioned. Also, they feel more responsible for their communities than for their patients. Thus, many physicians reject training opportunities, which may compete with the community and family time they are expected to engage in.

Some particularities exist in the manner that physicians in Zanzibar assume their responsibility over patients. As the chief of the Emergency Medicine Department commented during an interview, "In a rather small medical community, there is a deep fear of being poorly judged by their peers in case the patient dies or suffers from a complication." Moreover, among religious physicians, the fear of altering the divine plan designed for certain patients exists.

\section{Discussion}

International collaboration programs face two major challenges: ${ }^{18}$ first, the enormous patient load most African hospitals encounter, which includes urgent neurosurgical conditions such as pediatric hydrocephalus and cranial and spinal trauma; ${ }^{24}$ and second, the need to transmit high-quality neurosurgical education to local surgeons. In the last decade, several ICPs have started to collaborate in developing neurosurgery in Tanzania, with remarkable results. These ICPs' shared vision has mobilized a network of global surgeons focused on sharing resources and expertise, training neurosurgeons, and stimulating research in East Africa.

For example, Madaktari Africa started training neurosurgeons in rural Tanzania in 2006. ${ }^{8}$ In Dar es Salaam, the Tanzania Neurosurgery Project from Weill-Cornell Medical College has organized visits by neurosurgical fellows from overseas, weekly telecast conferences with local surgeons, and a yearly Neurotrauma Meeting, currently one of the most important scientific forums for neurosurgery in East Africa. ${ }^{11,23}$ Finally, with a different approach, the Duke East Africa Neurosurgery Program, spearheaded by Dr. Michael Haglund, has been successfully training neurosurgeons in Kampala, Uganda. Since 2015, they have also been partnering with the Mbarara Hospital, located in the Southwest region of the country. ${ }^{9}$

The existence of these ICPs and their mutual support 
and collaboration ameliorate the current status of neurosurgery in East Africa and favor a more dynamic and connected East African neurosurgical community.

\section{Considerations When Designing an ICP: The Integrative Model}

We strongly encourage all ICPs to conduct their operations under the patronage and support of larger entities such as the FIENS, WHO, or World Bank (www.worldbank. org) and to partner with existing African networks, such as the College of Surgeons of East, Central and Southern Africa (COSECSA; www.cosecsa.org), the Continental Association of African Neurological Societies (CAANS), and the Pan-African Association of Neurological Sciences (PAANS), as well as the local health authorities.

We believe that the utilization of local resources is the key to efficiency when designing an ICP. Also, sustainability requires consideration of the local social and cultural perspectives. Failure to consider such factors can lead to progressive discontent among local practitioners, resistance to new initiatives, and interpersonal distancing. Moreover, the ICP methodology needs to keep realistic expectations about the degree to which local practitioners are willing to change their practice. ${ }^{22}$

Intervention by the foreign team needs to be conducted with caution and respect, aiming to guide local physicians toward safer practices, while avoiding dominance, condescension, or the perpetration of deleterious cycles of dependence. Introducing smaller, progressive changes over time has true potential to improve the local situation, instead of major policy changes that threaten the status quo. Figure 4 summarizes the integrative model that we propose for an efficient ICP.

\section{Impact of the NEDF}

The NEDF has operated in Zanzibar since 2004. The main objectives of this ICP are to provide neurosurgical care to the Zanzibar population and to bring neurosurgical education and train surgeons. Since 2008, 129 ISCs have attended to Zanzibar's demand for neurosurgical care. Over 597 volunteers have been deployed, and thousands of neurosurgical procedures have been performed. Since 2016, more than 1000 patients have undergone neurosurgery at the NEDI, and 9169 outpatients have received counsel and ambulatory care. Before the arrival of the NEDF, the management of pediatric hydrocephalus was available only on Tanzania's mainland, and only infants from rich families could afford a delayed flight for treatment. Most pediatric patients would die before receiving neurosurgical care. However, since 2014, 592 ventriculoperitoneal shunts have been implanted at the NEDI, neuroendoscopic procedures are now locally available, and spina bifida repair is successfully performed by local surgeons.

By 2013, the NEDI was built thanks to private efforts under the direction of Paul $\mathrm{H}$. Young, José Piquer, and Mahmood M. Qureshi and led to a more dynamic relationship between NEDF and MMH. The NEDI received full endorsement by the Ministry of Health. The MMH personnel were hired at the NEDI, and the general maintenance of the building became the responsibility of the administrative department at MMH. The recruitment of local medical officers allowed the NEDF to have accurate insight into the most prevalent pathologies, the state of the management of trauma, and the overall ways into the Administration Department and the Ministry of Health of Zanzibar. These medical officers were fast-track trained to address neurosurgical emergencies in between ISCs.

One of the particularities of the NEDF as an ICP is that when it started operating in the MMH surgical theaters, no neurosurgical care was available in the region. Before the NEDF's presence in Zanzibar, patients had to be transported by air or water to the closest neurosurgical center on mainland Tanzania.

Because of the prior absence of neurosurgeons in Zanzibar, the impact of the NEDF has been an unprecedented positive change for the health of the population. For example, in 2006 , only 4 cases of severe TBI were reportedly admitted to the MMH's ICU, none of which received surgical treatment or survived after the 5th day of admission. Presumably, many other cases reached the hospital that year, but none were admitted to the ICU, likely due to the absence of guidelines or experience in the management of TBI. In contrast, in 2015 alone, 129 patients were admitted to the MMH's ICU with a diagnosis of severe TBI. Of these patients, 42 underwent surgery with a mortality rate of $32.5 \%$. Moreover, in 2017, almost $50 \%$ of the trauma surgeries were performed by local medical officers.

The operations of the NEDF have drawn the attention of intensivists, rehabilitation physicians, and other local specialists who, as part of the NEDF-MMH partnership, are now also receiving specific training and support through other medical branches of the NEDF.

The involvement of the NEDF in neurosurgical education is multilayered: 1) At the local level, the NEDF trains local surgeons and nurses through short-term volunteers (i.e., ISCs) and long-term volunteers (i.e., NCs), who develop daily academic activities. 2) At the continental level, the NEDI is accredited by COSECSA to formally train neurosurgeons. This endorsement facilitates the organization of international courses and meetings for trainees from East Africa. This allows connecting them with a global network of neurosurgical institutions, providing opportunity for trainees to benefit from participation in international meetings and enhance their clinical research skills. $^{5-7}$ 3) At the international level, the NEDF has promoted agreements between the RGZ and foreign training centers. Currently, two Zanzibari neurosurgeons are being formally trained in Egypt and in Cuba.

Progressively, the administrative structure for a selfsufficient neurosurgical center is being designed and implemented. Basic management protocols are being established, and local nurses and medical officers are becoming responsible for visiting patients, preparing the surgical list, and providing the NEDI with basic supplies. Education is the key element in the NEDF's approach to a sustainable training of neurosurgeons in Zanzibar. The NEDF will keep organizing ISCs, developing academic activities such as courses and symposiums, and enriching the neurosurgical literature on East Africa. ${ }^{12,18-20}$

The NED philosophy emphasizes that respect and consideration of social and cultural differences promote equality among foreign and local teams, protecting this 


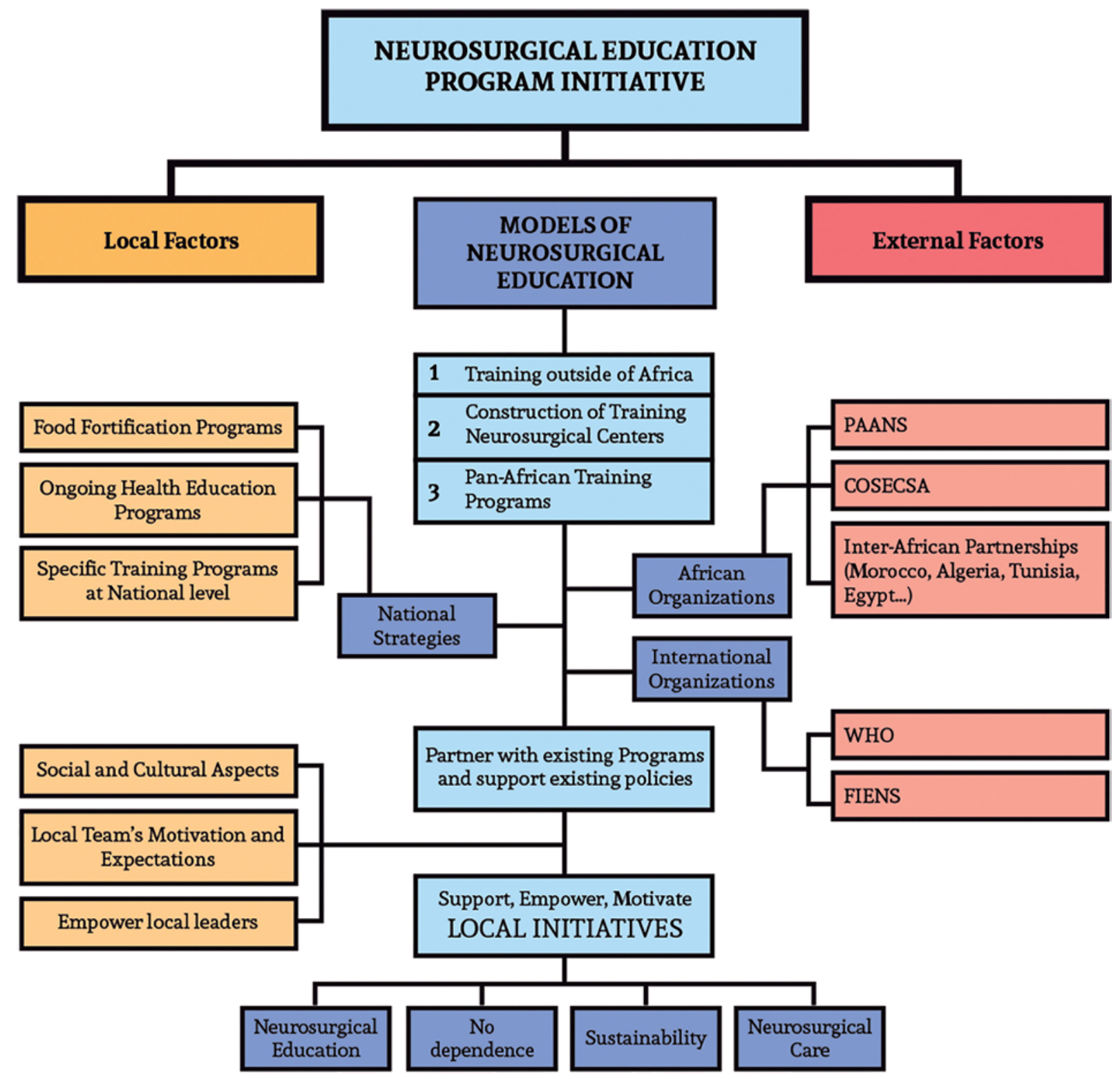

FIG. 4. International collaboration program integrative model. The flowchart describes all major elements in the conception of an ICP. The central cells (blue) describe (top to bottom) the sequential steps, options, and items that should be considered when designing an ICP. Local (yellow) and external (red) factors play important roles throughout the design and execution process, ultimately leading to effective neurosurgical education and care, sustainability, and nondependence.

ICP from dependency cycles and promoting neurosurgical care in Zanzibar as the responsibility of local practitioners. The NED volunteers actively seek to empower local initiatives and bring the necessary tools for them to take shape. The NEDF's objectives include leaving the management of the NEDI entirely in the hands of local practitioners in the future. From that point, the NEDF will act as a consultant entity, with less administrative responsibilities and possibly fewer ISCs per year.

\section{Conclusions}

International collaboration programs should balance local challenges and opportunities in order to effectively promote the development of neurosurgery in East Africa. Support and endorsement should be sought to harness shared resources and experience. Determining the caregiving and educational objectives within the logistic, administrative, social, and cultural framework of the target hospital is paramount to success.

\section{Acknowledgments}

We would like to thank Ms. Stephanie Leidinger for her support and contributions to the artwork in this study.

Dr. Leidinger was supported by a neurosurgery fellowship/grant provided by the NED Foundation. 


\section{References}

1. Amory D: The politics of identity on Zanzibar [thesis]. Stanford, CA: Stanford University, 1994

2. Babu AM: Zanzibar and the future. Change 2:28-33, 1994

3. Caputo R: Swahili coast: East Africa's ancient crossroads. National Geographic 200:104-119, 2001

4. Dempsey KE, Qureshi MM, Ondoma SM, Dempsey RJ: Effect of geopolitical forces on neurosurgical training in SubSaharan Africa. World Neurosurg 101:196-202, 2017

5. El-Fiki M: African neurosurgery, the 21st-century challenge. World Neurosurg 73:254-258, 2010

6. El Khamlichi A: African neurosurgery: current situation, priorities, and needs. Neurosurgery 48:1344-1347, 2001

7. El Khamlichi A: African neurosurgery. Part II: current state and future prospects. Surg Neurol 49:342-347, 1998

8. Ellegala DB, Simpson L, Mayegga E, Nuwas E, Samo H, Naman N, et al: Neurosurgical capacity building in the developing world through focused training. J Neurosurg 121:15261532,2014

9. Fuller A, Tran T, Muhumuza M, Haglund MM: Building neurosurgical capacity in low and middle income countries. eNeurologicalSci 3:1-6, 2015

10. Jhpiego: Zanzibar opens the first neurosurgery unit. Jhpiego. org. November 2, 2015. (https://www.jhpiego.org/successstory/zanzibar-opens-the-first-neurosurgery-unit/) [Accessed August 1, 2018]

11. Kahamba JF, Assey AB, Dempsey RJ, Qureshi MM, Härtl R: The second African Federation of Neurological Surgeons course in the East, Central, and Southern Africa region held in Dar es Salaam, Tanzania, January 2011. World Neurosurg 80:255-259, 2013

12. Leidinger A, Piquer J, Kim EE, Nahonda H, Qureshi MM, Young PH: Treating pediatric hydrocephalus at the Neurosurgery Education and Development Institute: the reality in the Zanzibar Archipelago, Tanzania. World Neurosurg [epub ahead of print], 2018

13. Lodhi AY: National language, culture and identity: the role of Kiswahili in the context of Zanzibar, in Proceedings of the International Conference on the History and Culture of Zanzibar. London: James Currey, 1992

14. Meara JG, Leather AJ, Hagander L, Alkire BC, Alonso N, Ameh EA, et al: Global Surgery 2030: Evidence and solutions for achieving health, welfare, and economic development. Surgery 158:3-6, 2015

15. Mitchell KB, Giiti G, Kotecha V, Chandika A, Pryor KO, Härtl R, et al: Surgical education at Weill Bugando Medical Centre: supplementing surgical training and investing in local health care providers. Can J Surg 56:199-203, 2013

16. National Bureau of Statistics: 2012 Population and Housing Census. Dar es Salaam, Tanzania: Ministry of Finances of Tanzania, 2014

17. Park KB, Johnson WD, Dempsey RJ: Global neurosurgery: the unmet need. World Neurosurg 88:32-35, 2016
18. Piquer J, Qureshi MM, Young PH: Impact of mobile endoscopy on neurosurgical development in East Africa. World Neurosurg 73:280-284, 2010

19. Piquer J, Qureshi MM, Young PH, Dempsey RJ: Neurosurgery Education and Development program to treat hydrocephalus and to develop neurosurgery in Africa using mobile neuroendoscopic training. J Neurosurg Pediatr 15:552-559, 2015

20. Qureshi MM: Humanitarian neurosurgery: mission in Zanzibar. FIENS.org. (http://www.fiens.org/humanitarianneurosurgery-mission-in-zanzibar/) [Accessed August 1, 2018]

21. Saleh MA: Tolerance: principal foundation of the cosmopolitan society of Zanzibar. Zaima Media Network. March 11, 2015. (https://zanzibardaima.net/2015/03/11/toleranceprincipal-foundation-of-the-cosmopolitan-society-ofzanzibar/) [Accessed August 1, 2018]

22. Spanu F, Piquer J, Panciani PP, Qureshi MM: Practical challenges and perspectives for the development of neurosurgery in a peripheral East African Hospital during a one-volunteer midterm mission. World Neurosurg 111:326-334, 2018

23. Wait SD, Härtl R: Multi-institutional American team teaches neurosurgery in underserved Tanzania. World Neurosurg 73:610-611, 2010

24. Wilson DA, Garrett MP, Wait SD, Kucia EJ, Saguda E, Ngayomela I, et al: Expanding neurosurgical care in Northwest Tanzania: the early experience of an initiative to teach neurosurgery at Bugando Medical Centre. World Neurosurg $77: 32-38,2012$

\section{Disclosures}

The authors report no conflict of interest concerning the materials or methods used in this study or the findings specified in this paper.

\section{Author Contributions}

Conception and design: Leidinger. Acquisition of data: Leidinger. Analysis and interpretation of data: Leidinger, Extremera, Kim. Drafting the article: Leidinger, Extremera, Kim. Critically revising the article: Leidinger, Extremera, Kim, Qureshi, Young. Reviewed submitted version of manuscript: Leidinger, Extremera, Kim. Approved the final version of the manuscript on behalf of all authors: Leidinger. Administrative/technical/material support: Leidinger, Piquer. Study supervision: Qureshi, Piquer.

\section{Correspondence}

Andreas Leidinger: Neurosurgery Education and Development Foundation, Valencia, Spain. andreas_leidinger@hotmail.com. 